\title{
Ultrasound Mediated Drug Delivery for the Management of Oral Diseases in Near Future
}

\author{
Himanshi Tomar*, Trishna Saikia and Vijeev Vasudevan \\ Department of oral medicine and radiology, Rajiv Gandhi University of Health sciences, India
}

*Corresponding author: Himanshi Tomar, Department of oral medicine and radiology, Rajiv Gandhi University of Health sciences, Bengaluru, Karnataka, India

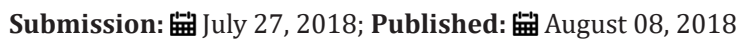

\begin{abstract}
Since 1980, Oral transmucosal drug delivery (OTDD) system is used in the treatment of systemic diseases. Despite of having various advantages of OTDD over the conventional oral drug delivery system like the ease of use and accessibility, surpassing the first pass metabolism etc., this method is still an emerging system due to relatively lower number of drug formulations available and anatomical barriers present in the oral cavity especially saliva. Ultrasound is one of the commonly used diagnostic tool and have many therapeutic applications in present scenario. Nowadays it is frequently used as an enhanced trans-epithelial drug delivery system which is based on the principle of transient cavitation that leads to the formation of microbubbles which in turn helps in the penetration of molecules into the permeable tissues. For the treatment of oral diseases, ultrasound is used in as local delivery of cancer chemotherapy, management of topical medication resistant inflammatory diseases, treatment of salivary gland injury and as a platform for gene therapy. This review explains the clinical application of ultrasound mediated transmucosal drug delivery in management of oral and systemic diseases.
\end{abstract}

Keywords: Ultrasound mediated drug delivery; Sonoporation; Gene therapy; Low frequency ultrasound

Abbreviations: OTDD: Oral Transmucosal Drug Delivery; LFU: Low Frequency Ultrasound; EGFR: Anti-Epidermal Growth Factor; Ca: Carcinoma; FAB: Fragment Antigen Binding; OSCC: Oral Squamous Cell Carcinoma; DOX: Doxorubicin; MBs: Micro-Bubbles; MTOR: Mammalian Target of Rapamycin; EGFR-MBs: Anti-Epidermal Growth Factor Antibody Conjugated Micro-Bubbles; IV: Intravenous; DNA: Deoxyribonucleic Acid; AQP1: Aquaporin-1; IL: Interleukin; NF-KB: Nuclear Factor Kappa B; ODN: Oligo De-Oxynucleotide; ICAM: Intercellular Adhesion Molecule; TNF: Tumor Necrosis Factor

\section{Introduction}

An efficacious drug delivery system is as important as a potent drug selection for an effective therapeutic outcome [1]. Oral transmucosal drug delivery (OTDD) is defined as the administration of pharmaceutically active agents through the oral mucosa to achieve systemic effects [2]. This conception of drug delivery system is used since many years and is successfully used as an alternative to the other oral or injectables drug forms for the treatment of systemic diseases [3]. Drugs which are topically used by the dermatologists for the treatment of skin diseases are currently the drug of choice for the treatment of oral mucosal diseases as there are few formulations designed for this purpose which can be used in the oral environment as the drug in most of the cases is washed off and the bioavailability is decreased due to the presence of saliva. So to achieve a standard therapeutic level, dosage of drug is raised and is administered repeatedly [4]. Numerous review articles have outlined the advantages of OTDD system over the conventional drug delivery system which includes ease of use, ease of accessibility, avoidance of first pass metabolism, less chances of systemic adverse drug reaction and a small area of mucosa is targeted $[2,5]$. In recent years the oral, nasal and vaginal mucosa has been used as way in for the drug delivery.
Low frequency ultrasound (LFU) is one of the most explored methods under OTDD system in recent years and its application as an enhanced trans-epithelial drug delivery system is well- known [5]. Ultrasound is a longitudinal pressure wave with a frequency above the audible range $(>20 \mathrm{kHz})[6]$. Sonophoresis is defined as the transport of drugs through the skin and into the soft tissue during or following the influence of an ultrasonic perturbation. Fellinger and Schmid were the first to use Ultrasound clinically as Transdermal drug delivery system for the treatment of poly-arthritis with hydrocortisone. Ultrasound frequencies used in the medicine vary from $20 \mathrm{KHz}$ to $16 \mathrm{MHz}$ out of which lower and medium range ultrasound frequencies are mostly used as ultrasound mediated drug delivery system due to higher cavitational effects [7]. The higher frequencies of ultrasound are used for diagnostic imaging, physiotherapy and pulverization of kidney and gallbladder stones $[1,8]$.

\section{Discussion}

\section{Physiology of the oral mucosa and saliva}

Oral mucosa in humans is constituted of stratified squamous epithelium and connective tissue. Both the components are 
separated by basement membrane [9]. Basal keratinocyte layer next to basement layer repairs and replenishes the epithelium by proliferation. Supra basal cells are present superficially to this layer and are partially differentiated. Oral cavity consists of regions which are keratinized and the superficial layer of epithelium is composed of differentiated keratinocytes which are shed after the desquamation [10]. Oral mucosa is a barrier for all the extrinsic material from environment that enters the soft tissues and helps in retaining the tissue fluids. The middle and the lower one third layers of epithelium are the main barriers for the permeation of extrinsic material into the soft tissues [4]. Oral cavity is constantly bathed in saliva due to which saliva can decrease the time of mucosal exposure to formulations by the dilution of drug in oral cavity and by the action of swallowing as drug can be cleared from oral cavity and reaches into gastrointestinal tract. Saliva however can also be advantageous as it provides a highly hydrated medium through which dissolved formulations can be distributed around the whole oral mucosa [11].

\section{Advantages and disadvantages of OTDD}

Delivery of therapeutics via oral mucosa has several advantages and disadvantages. Drugs are well tolerated and self-administered by the patient, easy accessibility of oral mucosa with rapid repair of any tissue injury, less systemic side effects as shorter recovery time, efficient delivery of drug as less quantity of drug is wasted or lost anywhere in body and fewer Langerhans cells in oral mucosa is responsible for reduced risk of drug allergy as compared to Transdermal route. Disadvantages of this route includes formulating drugs or delivery system that can permeate through the mucosal barrier easily, can overcome the enzymatic degradation of peptides and proteins, taste should be acceptable and should be easily administered $[4,12]$.

\section{Biological effects of ultrasound}

Cavitational effects: Cavitation may be defined as the formation of bubbles in an insonated medium due to ultrasound induced pressure changes. Cavitation is divided into two categories 1) Inertial, 2) Stable. In inertial cavitation depending on the ultrasound frequency bubble grows rapidly then after reaching a certain diameter, it collapses. In stable cavitation, there is a formation of stably oscillating bubbles which collapses due to Sono-chemical reactions in extreme conditions of temperature above 5000K [13] and pressure of 300bar [14]. Shock waves which are produced due to collapse of bubbles cause structural shifts to the surrounding tissues by microjets. Microjets are formed as a result of convection due to non- symmetrical bubble collapse. Threshold intensity is required for cavitation to occur and is directly proportional to the cavitational effect. Various factors that increase the cavitational effect or threshold intensity are increasing Ultrasound frequency, medium viscosity, ambient pressure, surface tension and ion concentration. Factors that decrease the cavitational effect are increase in medium temperature and gas content [15]. Usage of ultrasound contrast agents, low frequency ultrasound (LFU), gassy fluids and surfactants also increases the cavitational threshold intensity [1].

Thermal effects: Ultrasound increases mediums temperature by absorption of the sound waves and

thermal effect is directly propotional to the mediums absorption coefficient. Ultrasound intensity, frequency and exposure time also varies directly with the thermal effect of ultrasound [15].

Acoustic streaming effects: Acoustic streaming is the development of unidirectional flow of current in a fluid generated by sound waves and is occurred by ultrasound reflections and other distortions caused by wave propagations. Oscillations produced by the cavitational bubbles increases the acoustic streaming effect thus affecting the adjacent tissue structures by the development of shear stresses caused due to streaming velocities. Micro-streaming is defined as an induction of swirls of flow from an oscillating source and it alters permeability of membranes and increases cellular activity [16].

\section{Application of LFU in management of oral Diseases}

Local delivery of cancer chemotherapy: For the management of head and neck cancer, the LFU is used as a means to deliver the targeted chemotherapeutic agent by depositing high levels of drugs into the tumor affected site directly thereby increasing the efficiency of drug delivery which leads to the equivalent or superior therapeutic effect as compared to traditionally administered systemic drugs. However this method may have limited application as it is not used as a first line of treatment for head and neck tumors. But Sonophoresis shows effective transmucosal delivery of drugs so local drug delivery of drugs certainly seems possible [5]. An in vitro study was performed in which Sonoporation was used as a specific drug delivery system for squamous cell carcinoma with anti-epidermal growth factor (EGFR) antibody in which marked growth inhibition of Ca 9-22 cells was seen after the administration of lower dose of Bleomycin by sonoporation with the anti EGFR antibody. Scanning electron microscopic analysis showed surface deformation of Ca 9-22 cells and increased population of apoptotic cells. On administration of low dose of Bleomycin with Fab fragment of anti EGFR antibody. These results show that the treatment of oral squamous cell carcinoma is possible by administering drugs into cells by novel application of Sonoporation along with the Fab fragment [17].

In another study the synergistic effect of curcumin microemulsions and LFU stimulation on the Oral squamous cell carcinoma cell lines (OSCC-4 and OSCC-25) were tested. The results showed cyto-toxic effects in microscopic imaging on brief exposure to curcumin containing micro-emulsions with the additions of ultrasound especially on OSCC 25 cells as they showed ruptured and damaged cells after treatment [18]. An in vitro study demonstrated the effectiveness of Doxorubicin (DOX) delivered into three different tumor cell lines by Sonoporation as LFU increases the DOX auto-fluorescence signal and resulted in remarkable decrease in cell viability. Later on in-vivo study was performed to verify antitumor effects of DOX using mice which 
exhibited systemic lymphadenopathy and some peripheral lymph nodes reaching $10 \mathrm{~mm}$ in diameter. The results showed high cytotoxic effects followed by intracellular uptake of DOX along with Sonoporation that leads to extra-vasation of DOX from lymphatic endothelia and penetration into tumor tissues. Their findings showed inhibited tumor growth and diminished blood vessels in affected lymph nodes with low systemic toxic effects of DOX. Above mentioned study supports the usage of Sonoporation as drug delivery system for treating metastatic lymph nodes located outside the dissection area as well as it also highlights the, yet to be studied option in which LFU mediated drug delivery of radiosensitizing chemotherapy can be used concomitantly with radiation therapy to avoid systemic toxic effects of agents like Cisplastin [19].

Another study reported effective intracellular drug delivery to human gingival squamous carcinoma Ca 9-22 cells by Sonoporation with microbubbles (MBs). This study was first performed in vitro in which they develop anti-epidermal growth factor receptor (EGFR) antibody - conjugated MBs and assessed their effect along with Sonoporation and Bleomycin on Ca9-22 cells by flow cytometry and Hoechst staining which showed a strong cytotoxic effect on Ca 9-22 cells and increased number of apoptotic cells. Further an in vivo study was performed on murine squamous cell carcinoma model in which Bleomycin delivery by sonoporation with EGFRMBs showed remarkable anti-tumor activity. Together, their results states that EGFR- MBs and Sonoporation treatment could be novel drug targeting modality for oral squamous carcinoma chemotherapy treatment due to their increased efficacy and specificity for intracellular drug uptake [20].

Management of topical medication resistant inflammatory disease: Sonophoresis can be useful in cases where there is requirement of high tissue concentration of anti-inflammatory agent such as steroids or MTOR, especially where systemic therapy is undesirable and cases that are resistant to conventional topical therapy [5]. A study reported the successful treatment of ulcerative lichen planus which was resistant to topical treatment by using ultrasound instrument in form of vaginal probe coupled with Solmuderol soaked gauges [21]. Interestingly, the ultrasound instrument used in this study was designed for the oral health market (Dentaphor II), but no longer appears to be available [5] Another study described the role of LFU as a novel drug delivery system due to its enhanced trans-mucosal drug delivery through Gastro-intestinal mucosa [22]. Conceptually, the probes described in both the studies were very similar and demonstrates proof of concept with respect to the application of Sonophoresis for management of local disease [5].

1.1.1.Treatment of salivary gland injury: Sonophoresis can be useful in treatment of radiation induced salivary gland injury which is a chronic complication of radiation therapy to treat head and neck cancer. Amifostine is an effective free radical scavenger, so is used to reduce the radiation induced salivary gland injury by daily intravenous infusion given in close proximity to each fraction of radiation which is inconvenient for the patients and is not a common procedure followed in daily practice and radiation oncology. Along with those side effects of Amifostine that includes nausea, vomiting and hypotension. So a more direct route would be beneficial for patient. If a suitable platform could be designed, Sonophoresis can be used in conjunction with topical formulations and other cyto-protective drugs targeting the salivary glands might provide targeted doses of drugs directly to gland and can serve as an alternative to IV administration of drug [5].

Platform for gene therapy: Many studies have been performed till now by using Sonophoresis as a platform for gene therapy. A study was performed in which salivary gland secretions was increased by adeno viral mediated transfer of Aquaporin-1 C DNA to irradiated rat salivary glands [23]. Another study used gene therapy with intraoral Manganese superoxide dismutase plasmid/liposome coupled with Amifostine to mitigate radiation induced salivary gland dysfunction. Gene transfer using a viral vector is challenging concept as the effect is transient and it can elicit a host immune response in patients with chronic disease [24]. To overcome these challenges, ultrasound was used successfully to replace the viral vector in delivering AQP1 to porcine irradiated glands. Autoimmune diseases induced salivary gland dysfunction like Sjogrens syndrome was treated by Ultrasound assisted gene therapy in which IL-17 receptor and Fc together known as fusion protein was delivered to Sub-mandibular glands of mice. Results showed decreased SS autoantigen levels in mice [25].

Microbubbles as US contrast agents: A study was done to investigate the effect of ultrasound microbubbles technique in Nuclear Factor Kappa B (NF-KB) decoy Oligodeoxy nucleotide (ODN) transfection in the gingival tissue in mice. The western blot analysis indicated protein expression levels of IL-1 $\beta$, IL-6 and ICAM-1 were lower as compared to control groups. So these results suggested that Ultrasound is an effective method for decoy transfection in periodontal tissues [26].

Future drugs: challenge of delivery: Treatment of many chronic oral conditions uses antibody based drugs (Rituximab and Infliximab), peptides, and novel macromolecular biological drugs. These drugs are easily destroyed in the gastrointestinal tract when given orally. Bioavailability of protein and peptide drugs is reduced by $95 \%$ by enzymatic activity of saliva, if delivered to oral mucosa through topical application. So the optimization of drug delivery is essential as it limits the usefulness of these drugs for treatment of chronic diseases including immune- inflammatory oral diseases. Physical properties and size of many protein and antibody- based biological agents, limits their penetration through oral mucosa so an efficient permeability enhancer or drug carrier system is necessary to enhance penetration. Therapeutic Anti-TNF $\alpha$ antibodies and other peptides which are used for the treatment of oral lichen planus and recurrent apthous stomatitis are related to risk of potentially serious systemic side effects on long term parental administration. However, if the topical drug delivery system is used for these agents to directly deliver these drugs across the oral mucosa to the site of disease could improve the treatment of these diseases by limiting the systemic side effects [4]. 


\section{Limitations}

For the treatment of oral mucosal diseases OTDD system by using sonoporation has shown promising results and effects. But few limitations regarding anatomy of oral cavity is an inherent challenge to effective OTDD. The limited surface area of oral cavity and the uncontrolled salivary flow is a challenge for the drug delivery system. The dose of drugs used via this route in in vitro models is not a representative of typical clinical doses. The detailed drug metabolism and tissue integrity in vitro models has not been explored in detail till date. Simulation of environment of oral cavity is not possible using the in vitro models. Regulatory requirements for OTDD are a significant burden on pharmaceutics. There is a lack of effective correlations between in vitro permeation testing and in vivo performance as compared to Transdermal delivery. Till date no specific drugs have been designed for buccal delivery. This route of drug delivery requires more productive algorithms and establishment of mechanistic knowledge to fulfill the proposition and thereby can serve as a prospect of future [2].

\section{Conclusion}

The application of LFU (Sonophoresis) to enhance transepithelial drug delivery is well established and it can serve as an alternative path to standard oral or injected drug formulations with the goals of avoiding first pass metabolism and enhancing ease of use. This route can be used over many financially and physiologically expensive biologics if further studies are performed showing its successful clinical application regarding its increased efficacy, avoidance of first pass metabolism and ease of use. This approach can be used as an alternative to conventional forms of therapy in the management of oral diseases, if it can overcome all limitations especially regarding the anatomy of oral mucosa and salivary control and deserves additional investigations.

\section{References}

1. Azagury A, Khoury L, Enden G, Kost J (2014) Ultrasound mediated transdermal drug delivery. Adv Drug Deliv Rev 72: 127-143.

2. Sattar M, Sayed OM, Lane ME (2014) Oral transmucosal drug deliveryCurrent status and future prospects. Int J Pharm 471(1-2): 498-506.

3. Madhav NV, Shakya AK, Shakya P, Singh K (2009) Orotransmucosal drug delivery systems: a review. J Control Release 140(1): 2-11.

4. Sankar V, Hearnden V, Hull K, Juras DV, Greenberg MS, et al. (2011) Local drug delivery for oral mucosal diseases: challenges and opportunities. Oral Dis 17(1): 73-84.

5. Sonis TS (2017) Ultrasound mediated drug delivery. Oral Dis 23(2): 135138.

6. Polat BE, Hart D, Langer R, Blankschtein D (2011) Ultrasound-mediated transdermal drug delivery: Mechanisms, scope, and emerging trends. J Control Release 152(3): 330-348.

7. Mitragotri S (2004) Sonophoresis: a 50-year journey. Drug Discovery Today 9(17): 735-736.

8. Mitrogotri S (2005) Healing sound: the use of ultrasound in drug delivery and other therapeutic applications. Nat Rev Drug Discov 4(3): 255-260.
9. Nanci A (2003) Ten Cate's oral histology-development, structure and function. Mosby: MO, USA.

10. Salamat MN, Chittchang M, Johnston TP (2005) The use of mucoadhesive polymers in buccal drug delivery. Adv Drug Deliv Rev 57(11): 16661691.

11. Maggi L, Segale L, Conti S, Ochoa ME, Salini A, et al. (2005) Preparation and evaluation of release characteristics of $3 \mathrm{Tab}$ Gum, a novel chewing device. Eur J Pharm Sci 24(5): 487-493.

12. Sudhakar Y, Kuotsu K, Bandyopadhyay AK (2006) Buccal bioadhesive drug delivery-a promising option for orally less efficient drugs. J Control Release 114(1): 15-40.

13. McNamara WB, Didenko YT, Suslick KS (1999) Sonoluminescence temperatures during multi-bubble cavitation. Nature 401: 772-775.

14. McNamara WB, Didenko YT, Suslick KS (2003) Pressure during sonoluminescence. J Phys Chem B 107(30): 7303-7306.

15. K. Suslick (1988) Ultrasound. It's Chemical, Physical, and Biological Effects. In: Kenneth SS (Ed.), VCH, New York, USA, pp. 1-336.

16. Baker KG, Robertson VJ, Duck FA (2001) A review of therapeutic ultrasound: biophysical effects. Phys Ther 81(7): 1351-1358.

17. Maeda H, Tominaga K, Iwanaga K, Nagao F, Habu M, et al. (2009) Targeted drug delivery system for oral cancer therapy using sonoporation. J Oral Pathol Med 38(7): 572-579.

18. Lin H, Thomas JL, Chen H, Shen CM, Yang WJ, et al. (2012) In vitro suppression of oral squamous cell carcinoma growth ultrasoundmediated delivery of curcumin microemulsions. Int J Nanomedicine 7: 941-951.

19. Kato S, Mori S, Kodama T (2015) A novel treatment method for lymph node metastasis using a lymphatic drug delivery system with nano/ microbubbles and ultrasound. J Cancer 6(12): 1282-1294.

20. Hirabayashi F, Iwanaga K, Okinaga T, Takahashi O, Ariyoshi W, et al. (2017) Epidermal growth factor receptor-targeted sonoporation with microbubbles enhances therapeutic efficacy in a squamous cell carcinoma model. PLoS ONE 12(9): e0185293.

21. Walsh DS, Dunn CL, Konzelman J, Sau P, James WD (1995) A vaginal prosthetic device as an aid in treating ulcerative lichen planus of the mucous membrane. Arch Dermatol 131(3): 265-267.

22. Schoellhammer CM, Schroeder A, Maa R, Lauwers GY, Swiston A, et al. (2015) Ultrasound- mediated gastrointestinal drug delivery. Sci Transl Med 7(310): 310-168.

23. Delporte C, O Connell BC, He X, Lancaster HE, O Connell AC, et al. (1997) Increased fluid secretion after adenoviral-mediated transfer of the aquaporin-1 cDNA to irradiated rat salivary glands. Proc Natl Acad Sci USA 94(7): 3268-3273.

24. Epperly MW, Carpenter M, Agarwal A, Mitra P, Nie S, et al. (2004) Intraoral manganese superoxide dismutase-plasmid/liposome (MnSODPL) radioprotective gene therapy decreases ionizing irradiation- induced murine mucosal cell cycling and apoptosis. In Vivo 18(4): 401-410.

25. Wang Z, Zourelias L, Wu C, Edwards PC, Trombetta M, et al. (2015) Ultrasound-assisted non-viral gene transfer of AQP1 to the irradiated minipig parotid gland restores fluid secretion. Gene Ther 22(9): 739749.

26. Yamaguchi H, Ishida Y, Hosomichi J, Suzuki JI, Hatano K, et al. (2017) Ultrasound microbubble-mediated transfection of $\mathrm{NF}-\kappa \mathrm{B}$ decoy oligodeoxynucleotide into gingival tissues inhibits periodontitis in rats in vivo. PLoS ONE 12(11): e0186264. 
Creative Commons Attribution 4.0 International License

For possible submissions Click Here

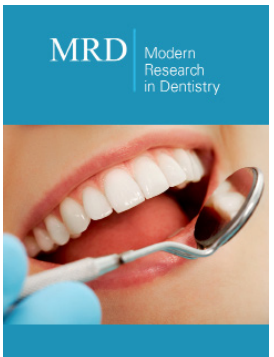

Modern Research in Dentistry

\section{Benefits of Publishing with us}

- High-level peer review and editorial services

- Freely accessible online immediately upon publication

- Authors retain the copyright to their work

- Licensing it under a Creative Commons license

- Visibility through different online platforms 